OPEN ACCESS

Edited by:

Raffaele De Caro,

University of Padua, Italy

Reviewed by:

Wei Seong Toh,

National University of Singapore,

Singapore

Monica Mattioli-Belmonte,

Marche Polytechnic University, Italy

*Correspondence:

Qian Chen

qian_chen@brown.edu

${ }^{\dagger}$ Present address:

Wenguang Liu,

Honghui Hospital, Xi'an Jiaotong

University, Xi'an, China

Meng Feng,

Department of Orthopedics Surgery,

The Second Affiliated Hospital of Xi'an

Jiaotong University, Xi'an, China

Specialty section:

This article was submitted to

Stem Cell Research,

a section of the journal

Frontiers in Cell and Developmental

Biology

Received: 14 June 2021

Accepted: 09 August 2021

Published: 06 September 2021

Citation:

Liu W, Brodsky AS, Feng M, Liu Y, Ding J, Jayasuriya CT and Chen $Q$ (2021) Senescent Tissue-Resident Mesenchymal Stromal Cells Are an Internal Source of Inflammation

in Human Osteoarthritic Cartilage. Front. Cell Dev. Biol. 9:725071. doi: 10.3389/fcell.2021.725071

\section{Senescent Tissue-Resident} Mesenchymal Stromal Cells Are an Internal Source of Inflammation in Human Osteoarthritic Cartilage

\author{
Wenguang Liu't, Alexander S. Brodsky2,3, Meng Feng't, Yajun Liu', Jing Ding', \\ Chathuraka T. Jayasuriya ${ }^{1}$ and Qian Chen ${ }^{1 *}$
}

' Department of Orthopedics, Rhode Island Hospital, Alpert Medical School of Brown University, Providence, Rl, United States, ${ }^{2}$ Department of Pathology and Laboratory Medicine, Rhode Island Hospital, Alpert Medical School of Brown University, Providence, RI, United States, ${ }^{3}$ Center for Computational Molecular Biology, Brown University, Providence, Rl,

United States

Human osteoarthritic cartilage contains not only chondrocytes (OACs), but also mesenchymal stromal cells (OA-MSCs), whose abundance increases during osteoarthritis (OA). However, it is not clear how OA-MSC contributes to OA pathogenesis. Here, we show that aging OA-MSC plays an important role in cell senescence, fibrosis, and inflammation in cartilage. Protein array analysis indicates that OA-MSC expresses pro-inflammatory senescence associated secretory phenotype (SASP) including IL-1 $\beta, I L-6, I L-8$, and CXCL1, 5, and 6, which play key roles in $\mathrm{OA}$ pathogenesis. OAC is a main recipient of the inflammatory signals by expressing receptors of cytokines. RNAseq analysis indicates that the transition from normal cartilage stromal cells (NCSCs) to OA-MSC during aging results in activation of SASP gene expression. This cell transition process can be recapitulated by a serial passage of primary OAC in cell culture comprising (1) OAC dedifferentiation into NCSClike cells, and (2) its subsequent senescence into pro-inflammatory OA-MSC. While $\mathrm{OAC}$ dedifferentiation is mediated by transcriptional repression of chondrogenic gene expression, OA-MSC senescence is mediated by transcriptional activation of SASP gene expression. We postulate that, through replication-driven OAC dedifferentiation and mesenchymal stromal cell (MSC) senescence, OA-MSC becomes an internal source of sterile inflammation in human cartilage joint.

Keywords: osteoarthritis, mesenchymal stem cell, cell senescence, cartilage, SASP

\footnotetext{
Abbreviations: OACs, osteoarthritic chondrocytes; NCSCs, normal cartilage stromal cells; OA-MSC, osteoarthritismesenchymal stromal cell; ECM, extracellular matrix; CHI3L1, chitinase-3-like-protein 1; RT-PCR, reverse transcriptasepolymerase chain reaction; SASP, senescence-associated secretory phenotype; RNAseq, RNA sequencing; GO, Gene Ontology; GSEA, Gene Set Enrichment Analysis; IPA, Ingenuity Pathway Analysis; EMT, epithelial-mesenchymal transition; CMT, chondro-mesenchymal transition; SACTAI, Senescence Associated Cell Transition and Interaction.
} 


\section{INTRODUCTION}

Osteoarthritis (OA) is an aging associated joint degenerativedisease affecting more than 300 million people worldwide. It is characterized by cartilage degradation, bone remodeling, and low-grade inflammation termed "inflammaging" (Coryell et al., 2020). However, the pathological mechanism of $\mathrm{OA}$ has not been well understood, which contributes to the lack of any FDA-approved drugs to modify $\mathrm{OA}$ pathogenesis. Although OA was not considered as an inflammatory disease historically, it has become more evident in recent years that the persistent long-term elevation of low-grade inflammation plays an important role in $\mathrm{OA}$ pathogenesis (Robinson et al., 2016). The sustained chronic production/presence of cytokines including TNF- $\alpha$, IL-1 $\beta$, IL- 6 , IL-8, and IL-17 and chemokines including CXCL1, 3, 5, and 6 and SDF-1 have been shown to be involved in the onset and progression of OA (Goldring, 2007). Cytokines and chemokines bind to their respective receptors on chondrocytes to trigger intracellular kinase signaling, stimulate the synthesis of matrix metalloproteinase (MMP), and promote matrix degradation and cell migration (Borzi et al., 2000). It was thought that the pro-inflammatory cytokines and chemokines come from intra-articular tissues outside of cartilage, such as synovium, fat pad, blood vessels, bone periosteum, and bone marrow (Xie and Chen, 2019). Cartilage has not been considered as a source of inflammatory cytokines and chemokines because of its lack of immune cells and blood vessels that produce such inflammatory factors.

The most common type of cell in articular cartilage is the chondrocyte. In adult humans, normal healthy articular chondrocytes are quiescent (Goldring, 2012). The collagen fibrils comprising Col II, XI, and IX are synthesized and laid down by chondrocytes during development (Mendler et al., 1989). The half-life of collagen is more than 100 years (Sivan et al., 2008), which favors a stable extracellular matrix (ECM) that carries out its structural functions. Metabolism of the quiescent chondrocytes is activated by injury and/or inflammation during post-traumatic OA and aging-associated OA (Goldring, 2012). OA chondrocytes increase the synthesis of ECM molecules as well as ECM degradative enzymes (Goldring, 2000b). It was thought that cartilage ECM turnover and remodeling depend on the balance of anabolism and catabolism in chondrocytes (Ragan et al., 2000). The pro-inflammatory environment such as the elevation of cytokines and chemokines in the joint tilts the balance toward catabolism during OA.

It has been demonstrated that articular chondrocytes undergo cell senescence during aging and/or injury (Ming et al., 2018; Chu et al., 2020). The concept of cell senescence was first proposed by Hayflick almost 60 years ago (Hayflick and Moorhead, 1961). He showed that through serial cell passaging and subculture, fetal lung fibroblasts ceased dividing and reached senescence (replicative senescence) (Hayflick and Moorhead, 1961). Senescent cells express molecular markers of cell senescence including $\mathrm{p} 16^{\mathrm{INK} 4 a}$, erode telomeres, and acquire the senescence associated secretory phenotypes (SASPs) (Coppé et al., 2011). The SASP contains aforementioned pro-inflammatory cytokines and chemokines as well as matrix degradative enzymes including MMPs (Tchkonia et al., 2013). It has been shown that human OA cartilage contains senescent chondrocytes that exhibit all the hallmarks of cell senescence including $\mathrm{p} 16^{I N K 4 a}$ and SASP (Martin and Buckwalter, 2003). While it is well established that serial passage of human primary chondrocytes induces dedifferentiation into a fibroblastic phenotype, it is not clear whether it also induces replicative senescence (Edith et al., 2019).

For a very long period, it was thought that chondrocytes were the only cell type in cartilage. In 2004 mesenchymal stromal cells (MSCs), which were also considered to be a kind of chondroprogenitor cell, were found in normal and OA human articular cartilage (Alsalameh et al., 2004; Dowthwaite et al., 2004). These MSCs can be isolated through their adhesion to fibronectin on a culture plate while chondrocytes do not (Alsalameh et al., 2004; Dowthwaite et al., 2004). They are positive for CD166 while chondrocytes are CD166 negative (Liu et al., 2020). They have low abundance in normal young cartilage (1\%), which is increased to $10 \%$ and more during OA pathogenesis (Alsalameh et al., 2004). However, it is not known how their abundance is increased and whether such increase contributes to $\mathrm{OA}$ pathogenesis. To answer these questions, we isolated MSCs from aging human OA cartilage (OA-MSC) (Jayasuriya et al., 2018) and from normal cartilage of young adults (NCSC) (Jayasuriya et al., 2019). In this study, we test the hypothesis that OA-MSCs are the senescent cells in cartilage whose abundance is increased during aging and $\mathrm{OA}$ pathogenesis. We provide molecular evidence that OA-MSCs are responsible for synthesizing the SASP that contributes to OA pathogenesis.

\section{RESULTS}

\section{Elevated Pro-inflammatory Protein Expression in OA-MSC}

To determine the pro-inflammatory protein profiles in human OA cartilage cells, we isolated two types of cells from cartilage, OAC and OA-MSC, using fibronectin-adhesion assay as described previously (Fellows et al., 2017; Jayasuriya et al., 2018). Flow cytometry analysis confirmed that the fibronectin adherent cells were CD166+ OA-MSC, and the fibronectin nonadherent cells were CD166- OAC (Jayasuriya et al., 2018). Since human OAC was prone to de-differentiation after multiple passages, only early-passage of primary OAC and OA-MSC were used for protein array analysis. Among 105 cytokines, chemokines, and other pro-inflammatory factors on the panel (Supplementary Table 1), a total of 19 proteins were detected in OA-MSC and OAC (Figures 1A,B). Among them, 18 were detected in OA-MSC (94.7\%) while $5(22.7 \%)$ were detected in OAC; 16 were up-regulated in OA-MSC (84.2\%) while 3 were up-regulated in OAC (15.8\%) (Figure 1B). These observations indicated that OA-MSC was the predominant type of cell that expressed pro-inflammatory factors in cartilage in terms of the number of cytokines and the extent of their protein levels. The pro-inflammatory factors up-regulated in OA-MSC comprised 
major cytokines and chemokines implicated for OA pathogenesis including IL-6, IL-8, IL-1Ra, CXCL1, CXCL5, CCL20, and IL-17A (Figure 1B).

To determine whether the expression of pro-inflammatory proteins in OA-MSC was associated with aging, we compared the protein profiles of OA-MSC from older OA patients with NCSCs (normal cartilage stromal cells) from young non-OA patients. Since the NCSC cells comprised only $1 \%$ of total cartilage cells (Alsalameh et al., 2004), the number of primary NCSCs was too low to extract sufficient amount of protein required for proteomic analysis. To overcome this difficulty, we generated cell lines of NCSC (Jayasuriya et al., 2019) and OA-MSC (Jayasuriya et al., 2018) using primary CD166+ stromal cells isolated from normal and OA cartilage, respectively. An OA-MSC cell line (OA-MSC2) and a NCSC cell line (NCSC3) were randomly chosen for protein array analysis. Among 105 inflammatory factors on the panel (Supplementary Table 1), a total 16 proteins were detected in OA-MSC and NCSC (Figures 1C,D). Among them, 15 were detected in OA-MSC (93.8\%) while 10 were detected in NCSC $(62.5 \%) ; 9$ were up-regulated in OA-MSC (56.3\%) compared to NCSC while 7 were up-regulated in NCSC compared to OA-MSC (43.8\%) (Figure 1D). Thus, the expression of proinflammatory factors of cartilage stromal cells was increased in the number of factors and the expression levels during aging process. The inflammatory cytokines up-regulated in $\mathrm{OA}$ MSC than NCSC included IL- $1 \alpha$, IL-1 $\beta$, and IL-8 (CXCL8), major SASPs that played important roles in OA pathogenesis (Coppe et al., 2010).

\section{Suppressed Chondrogenic Genes and Elevated SASP and Fibrosis Genes in OA-MSC}

To determine whether up-regulation of SASP protein expression occurs at the transcription level, we performed whole transcriptome RNA sequencing with the RNA purified from OAC, OA-MSC, and NCSC. Pairwise comparison was conducted with the 30 most significantly differentially expressed genes between OAC and OA-MSC (Figure 2A). Among them, 22 were down-regulated and 8 were upregulated in OA-MSC in comparison to OAC. The RNAseq analysis indicated that the chondrocyte marker CHI3L1 (chitinase-3-like-protein 1 or human cartilage gp-39) was the most significantly down-regulated genes in OA-MSC (Figure 2A, green arrow). This suggested that chondrogenic genes were suppressed in OA-MSC through down-regulation of their transcript levels. In addition, cartilage ECM markers including COL11A1 and PRG4 were up-regulated in OAC in comparison to OA-MSC (Figure 2A, green arrows). This indicated that $\mathrm{OAC}$ plays an anabolic role in synthesizing ECM in human OA cartilage. The up-regulated genes in OA-MSC included TNFRSF1B (TNFR2) (Figure 2A, red arrow), indicating that OA-MSC played a role in TNF- $\alpha$ signaling.

Pairwise comparison between NCSC and OA-MSC indicated that all the top 30 most significantly differentially expressed genes were up-regulated genes in OA-MSC (Figure 2B). This suggested a transcriptional activation during the cartilage MSC aging process. The most significantly up-regulated genes in OAMSC were SASPs including IL1B, IL6, CXCL1, CXCL5, CXCL6, CXCL8 (IL-8) (Figure 2B, red arrows). Since these are key inflammatory factors during $\mathrm{OA}$ pathogenesis, it suggested that OA-MSC play an important role in cartilage inflammation by synthesizing SASP. The mRNA levels of fibrosis markers in cartilage were also up-regulated in OA-MSC. They included fibroblastic ECM FN1, COL3A1, COL5A2, VCAN, and their crosslinking enzyme LOX (Figure 2B, brown arrows). This suggested that OA-MSC play an important role in cartilage fibrosis as well.

\section{SASPs Are Molecular Markers of OA-MSC}

We focused on analyzing four molecules highly expressed by OAMSC in the cell senescence and inflammation pathway: CXCL1, $I L 1 B, T N F R 2$, and $p 16^{I N K 4 a}$. RNAseq analysis indicated that two SASP molecules CXCL1 and $I L 1 B$ were highly expressed in OA-MSC (Figure 2C), suggesting that they were molecular markers of OA-MSC. TNFR2 mRNA was expressed at a moderate level in OAC and its expression level became higher in NCSC and OA-MSC (Figure 2C). The expression of cell senescence marker $p 16^{I N K 4 a}(P 16)$ was low in OAC, higher in NCSC, and the highest in OA-MSC (Figure 2C). To validate the RNAseq data, we purified RNA in primary OAC and OA-MSC isolated from human cartilage of multiple patients $(n=5)$. Real-time RT-PCR analysis demonstrated that the CXCL1 transcript was greatly up-regulated in OA-MSC than OAC $(p<0.01)$ (Figure 2D). Additional passages of primary OA-MSC in culture further increased the levels of CXCL1 (Figure 2D). While $I L 1 B$ mRNA was not detected in OAC, it was induced in OA-MSC $(p<0.01)$ (Figure 2D). Similarly, the mRNA levels of TNFR2 $(p<0.01)$ and P16 $(p<0.01)$ were significantly increased in OA-MSC than OAC, respectively. Passaging primary OA-MSC further increased the transcript levels of $I L 1 B, T N F R 2$, and P16, similar to that of CXCL1 (Figure 2D). These data suggest that the increase of the mRNA levels of the SASPs was associated with OA-MSC and its passages in culture.

To determine whether the increase of these OA-MSC markers was associated with aging, we compared their levels in the NCSC cells derived from a young patient and those of OAMSC derived from old patients. The CXCL1 and ILIB mRNA levels were greatly increased in OA-MSC compared to NCSC $(p<0.01)$ (Figure 2E). The mRNA levels of TNFR2 $(p<0.01)$ and P16 were increased in OA-MSC, respectively (Figure 2E). This suggested that the up-regulation of the OA-MSC markers was associated with the MSC aging process in cartilage. The increase of CXCL1 and IL1B expression in OA-MSC was also seen at the protein levels (Figure 1), and CXCL1 protein expression was also detected in NCSC. Western blot analysis demonstrated that, while the TNFR2 protein level was low in OAC, it was increased in OA-MSC especially at high cell passages (Figures 2F,G). Taken together the RNA and protein expression data, it suggested that the induction of 


\section{A}

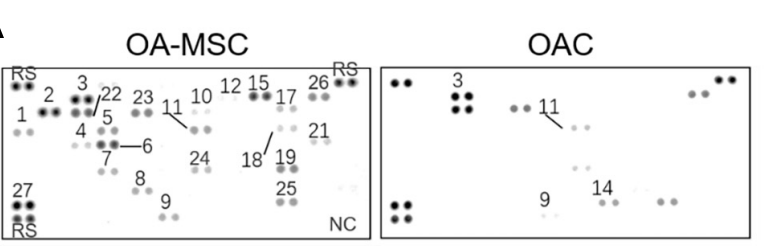

C

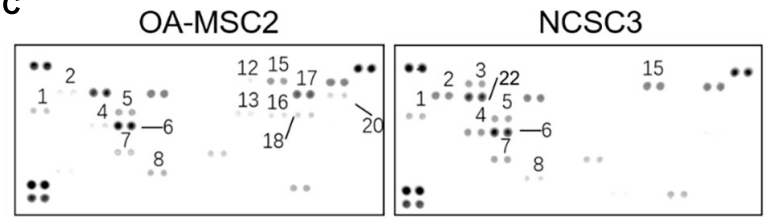

B

ID\#

2

15

6

19

5

5

9

7

17

21

4

18

10

12

8

11

24

25

23

26

3

22

14

\begin{tabular}{|c|c|}
\hline Protein & OA-MSC \\
\hline CXCL5 & $68.57 \pm 1.3$ \\
\hline CD26 & $41.62 \pm 1.23$ \\
\hline IL-8 & $39.35 \pm 1.13$ \\
\hline CCL20 & $22.13 \pm 0.73$ \\
\hline CD54 & $16.74 \pm 0.34$ \\
\hline CXCL1 & $12.42 \pm 0.32$ \\
\hline CD106 & $10.92 \pm 0.07$ \\
\hline $\mathrm{CCL} 2$ & $9.97 \pm 0.12$ \\
\hline GDF15 & $8.14 \pm 0.32$ \\
\hline IL-17A & $5.89 \pm 0.57$ \\
\hline IL-6 & $5.37 \pm 0.1$ \\
\hline IL-1 ra & $5.08 \pm 0.98$ \\
\hline FGF19 & $2.25 \pm 0.44$ \\
\hline Dkk1 & $0.81 \pm 0.15$ \\
\hline PTX3 & $11.56 \pm 0.51$ \\
\hline IGFBP-3 & $16.27 \pm 0.95$ \\
\hline MIF & $8.61 \pm 0.45$ \\
\hline uPAR & $17.46 \pm 0.21$ \\
\hline FGF2 & $24.62 \pm 0.9$ \\
\hline CD147 & $20.83 \pm 0.29$ \\
\hline CHI3L1 & $80.74 \pm 2.28$ \\
\hline CD105 & $33.93 \pm 1.2$ \\
\hline TSP-1 & \\
\hline
\end{tabular}

0 OAC

0

$$
0
$$

0

0

0

0

0

0

0

0

0

0

0

0

$0.96 \pm 0.18$

$4.88 \pm 0.02$

$3.01 \pm 0.44$

$14.51 \pm 0.49$

$22.87 \pm 0.02$

$21.63 \pm 0.61$

$133.2 \pm 2.5$

$104.8 \pm 5.74$
$10.99 \pm 0.02$

\section{Fold Change}
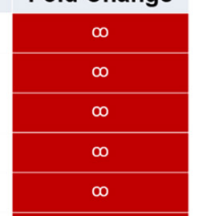

$\infty$

$\infty$

$\infty$

$\infty$

$\infty$

$\infty$

$\infty$

$\infty$

$\infty$

$\infty$

$\infty$

12.08

3.33

2.86

1.2

1.08

1.04

$-1.65$

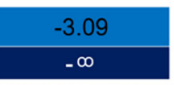

$-\infty-3 \quad 12 \infty$

.

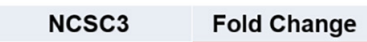

\begin{tabular}{|r|r|r|}
\hline 17 & GDF15 & $33.36 \pm 0.27$ \\
\hline 18 & IL-1ra & $3.58 \pm 0.68$ \\
\hline
\end{tabular}

Fold Change

CSF2

IL-1b

$3.01 \pm 0.66$

$1.88 \pm 0.21$

IL-1a

$1.44 \pm 0.24$

$0.44 \pm 0.62$

$7.4 \pm 0.12$

PTX3

IL-8

UPAR

\begin{tabular}{l|l}
$89.55 \pm 17.87$ & $63.84 \pm 14.7$ \\
\hline
\end{tabular}

$7.879 \pm 0.57$

CD105

FGF2

$9.73 \pm 0.58$

$43.27 \pm 0.23$

$24.62 \pm 0.55$

$22.46 \pm 0.21$

CD54

$10.95 \pm 0.21$

$11.62 \pm 0.1$

$24.55 \pm 0.06$

$22.49 \pm 0.21$

$5.43 \pm 0.2$

$7.75 \pm 0.33$

$11.85 \pm 0.21$

$23.3 \pm 0.36$

$14.36 \pm 0.21$

$20.14 \pm 1.02$

CXCL5

$1.47 \pm 0.86$

$1.21 \pm 0.14$

$12.29 \pm 0.82$

0

0

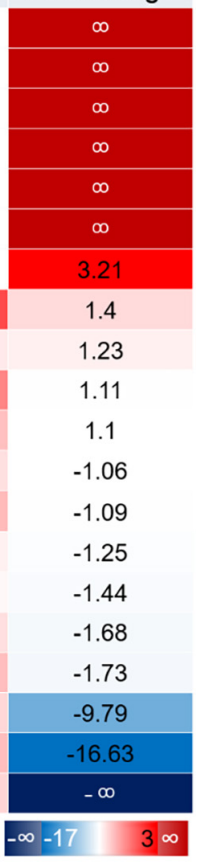

FIGURE 1 | Human cytokine protein array analysis. Differential protein expression analysis of cytokines and related proteins was performed using cell extracts of primary human cartilage cells OA-MSC and OAC (A,B), and cell extracts of human cartilage MSC cell lines OA-MSC2 and NCSC3 (C,D). (A,C) Cytokine protein signals were detected on the membranes of protein arrays. ID number of the protein was indicated. (B) Differential protein expression profiles of OA-MSC and OAC. The unit number of the mean spot pixel density signal was indicated for each detected protein in OA-MSC (third column) and OAC (fourth column). Red color reflects the protein expression level. The relative fold change of protein signals of OA-MSC vs. OAC was indicated in the fifth column. Red color indicates higher protein levels in OA-MSC while blue color indicates higher protein levels in OAC. (D) Differential protein expression profiles of OA-MSC2 and NCSC3. The unit number of the mean spot pixel density signal was indicated for each detected protein in OA-MSC2 (third column) and NCSC3 (fourth column). Red color reflects the protein expression level. The relative fold change of protein signals of OA-MSC2 vs. NCSC3 was indicated in the fifth column. Red color indicates higher protein levels in OA-MSC2 while blue color indicates higher protein levels in NCSC3.

SASP gene expression in OA-MSC occurred at both mRNA and protein levels.

\section{Cell Phenotype Transitions During Cell Aging and Senescence}

To identify the pathways connecting OAC, OA-MSC, and NCSC, we performed Gene Set Enrichment Analysis (GSEA) (Subramanian et al., 2005) with the RNAseq data of these three types of cells. GSEA revealed multiple pro-inflammatory pathways were activated in OA-MSC in comparison to NCSC and OAC. They include inflammatory response $(\mathrm{FDR}<0.001 ; \mathrm{FDR}=0.005), \mathrm{TNF} \alpha$ signaling via NFK $\mathrm{B}$ $($ FDR $<0.001 ;$ FDR $=0.003)$, and IL6 JAK STAT3 signaling
$(\mathrm{FDR}=0.006 ; \mathrm{FDR}=0.007)$ (Figures 3A,B). This suggested that the acquisition of the pro-inflammatory phenotype in OA-MSC compared to NCSC and OAC. GSEA revealed that the epithelial mesenchymal transition (EMT) gene set was enriched during transition from NCSC to OAMSC (FDR $<0.001)$ (Figure 3A), suggesting that the mesenchymal cell phenotype changes occurred during aging and senescence process.

\section{The OAC to OA-MSC Transition Involves Two Stages}

To analyze cell transitions among OAC, NCSC, and OAMSC, we performed volcano plot and Gene Ontology (GO) 


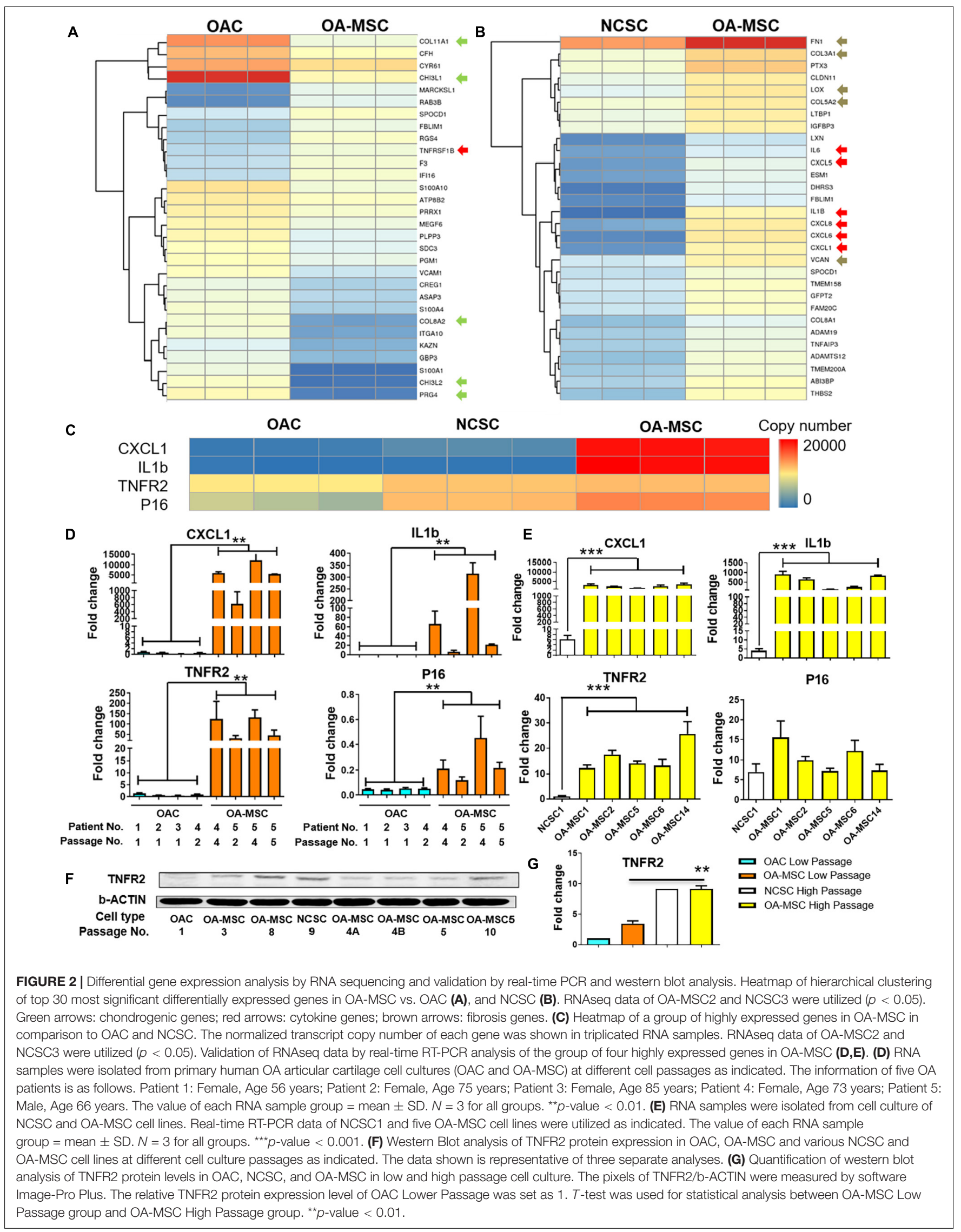




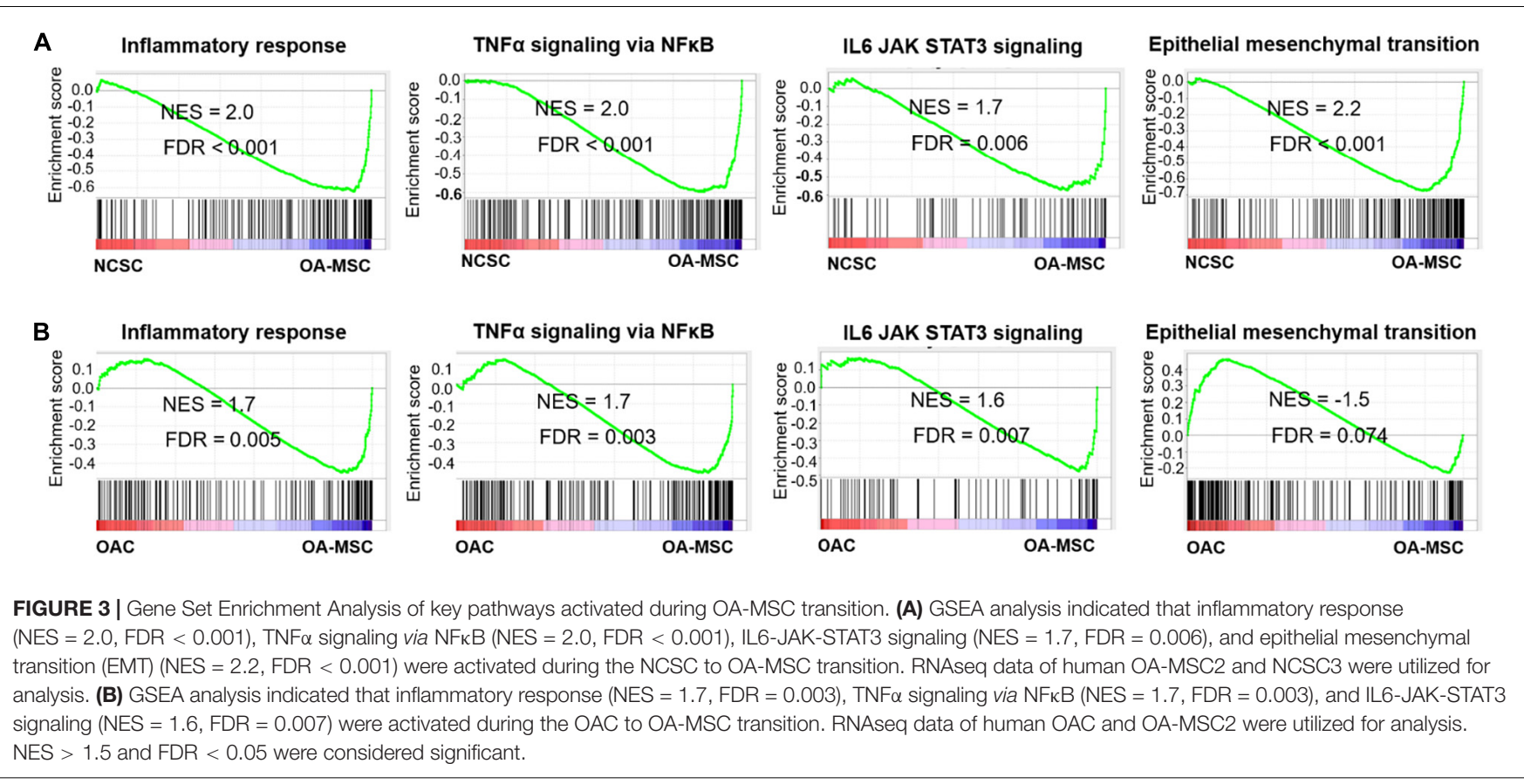

analysis. The volcano plot analysis demonstrated differential gene expressions in $\mathrm{OAC}$ and $\mathrm{OA}-\mathrm{MSC}$ (Figure 4A). GO enrichment analysis indicated that, among the top 20 gene sets that were significantly differentially expressed between OAC and OA-MSC, 16 were up-regulated in OAC while 4 were up-regulated in OA-MSC (Figure 4B). The up-regulated gene sets in OAC consisted of three categories: (1) ECM structural function (ECM binding, structural constituent, and mechanical properties); (2) ECM regulatory function (growth factor binding); and (3) cell receptor signaling (scavenger and cargo receptor activity and transmembrane receptor tyrosine kinase activity) (Figure 4B, OAC $>\mathrm{OA}$ MSC). The up-regulated gene sets in OA-MSC were (1) cytokine and growth factor activity, and (2) chemokine and growth factor receptor binding (Figure 4B, OAMSC > OAC). Thus, the OAC to OA-MSC transition involved (1) down-regulation of chondrogenic ECM and receptor gene expression and (2) up-regulation of cytokine/chemokine and receptor binding.

RNA sequencing analysis suggested that down-regulation of chondrogenic ECM and receptor gene expression occurred during the OAC to NCSC transition (Figures 4D,E). Volcano plot indicated that more genes were significantly downregulated in NCSC, suggesting a transcriptional repression during the transition of OAC to NCSC (Figure 4D). Among the down-regulated genes were chondrocyte markers CHI3L1, COL11A1, and PRG4 (Supplementary Figure 1, green arrows), the same down-regulated genes during the OAC to OA-MSC transition (Figure 2A). Consistent with this finding, GO enrichment analysis indicated that all top 20 gene sets differentially expressed between OAC and NCSC were down-regulated in NCSC (Figure 4E). They contained the same three categories of gene sets down-regulated during the OAC to OA-MSC transition (Figures 4B,E).

\section{OA-MSC Acquires Inflammatory and Fibrosis Gene Expression During Cell Aging}

RNA sequencing analysis suggested that up-regulation of cytokine/chemokine and receptor gene expression occurred during the NCSC to OA-MSC transition. The volcano plot analysis demonstrated that more genes were significantly up-regulated in OA-MSC than NCSC (Figure 4G), suggesting the OA-MSC transcriptome was activated during aging. GO enrichment analysis indicated that OA-MSC contained all top 20 up-regulated gene sets between NSCS and OAMSC (Figure $4 \mathbf{H}$ ). The up-regulated gene sets contained the same cytokine/chemokine gene sets upregulated during the OAC to OA-MSC transition (Figure 4B). In addition, the up-regulated gene sets also contained fibrotic ECM genes, consistent with the pair-wise RNAseq analysis (Figure 2B).

To identify the upstream regulators of the transitions among OAC, NCSC, and OA-MSC, we performed Ingenuity Pathway Analysis (IPA). The top five upstream regulators of the OAC to OA-MSC transition were (1) SMARCA4, a chromatin ATPase and transcription regulator; (2) TGFB1, a growth factor that activated catabolism in OA-MSC (Liu et al., 2020); (3) TNF, an inflammatory cytokine activating joint degeneration; (4) ESR1; and (5) estrogen receptor, critical regulators of estrogen signaling during OA pathogenesis (Figure 4C). These factors were involved in the OAC to NCSC transition (Figure 4F) and the NCSC to OA-MSC transition (Figure 4I). Two of these factors SMARCA4 and TGFB1 were 





common regulators. While ESR1 regulated the OAC to NCSC transition, estrogen receptor and TNF regulated the NCSC to OA-MSC transition. The unique regulators of the OAC to NCSC transition contained ERBB2 (a receptor protein kinase) and HNRNPA2B1 (a RNA binding and processing protein). TNF and $\mathrm{EZH} 2$ (a histone methyltransferase) were the top unique factors in the NCSC to OA-MSC transition, illustrating the role of inflammation and epigenetic regulation during MSC aging and senescence.

\section{Serial Passaging of OAC Induced Dedifferentiation and Recapitulated Cell Senescence and Inflammation Process}

To determine whether OAC could be transitioned into OAMSC, we cultured primary articular chondrocytes from OA patients through serial passaging. OAC were cultured under standard conditions recommended for chondrocytes (OAC medium) or mesenchymal stromal cells (OA-MSC medium) (Figure 5D). Both OAC and OA-MSC media contained identical components including high glucose DMEM, 10\% FBS, and antibiotics (Penn Strep). In addition, OA-MSC medium contained high energy sources including additional L-glucose, sodium pyruvate, and GlutaMAX. Flow cytometry analysis indicated that the percentage of CD166+ cells (MSC marker) increased during cell passagingg from the initial $2.9 \%$ at $\mathrm{P} 0$ 95.4\% at P5 (Figure 5A, $x$-axis). Cell morphology was also changed from the original cuboidal shape of chondrocytes (aquamarine arrows) to the long spindle shape typical of fibroblasts (gray arrows) during cell passages (Figure 5B). This fibroblast morphology was more evident during incubation in the OA-MSC medium. At P5, the senescent cell morphology (yellow arrows) of a large cell size with extended processes started to appear (Figure 5B). Flow cytometry confirmed more large-sized cells in the P5 culture (Figure 5A, $y$-axis). After $\mathrm{P} 5$, the cells reached full senescence and stopped proliferating. Flow cytometry also revealed that cells reached the CD166+ status faster when cultured in the OA-MSC medium (Figure 5A, $x$-axis).

Real-time RT-PCR analysis demonstrated that the markers of OA-MSC were induced by cell passaging (Figure 5C). The expression levels of these markers were higher when cells cultured in the OA-MSC medium than the OAC medium. Most interestingly, the pro-inflammatory cytokine/chemokine CXCL1 and CXCL8 $(I L-8)$ were not detected at P2 in the OA-MSC medium or during P2-P3 in the OAC medium. This suggested that these cells acquired their inflammation phenotype at P3 in the OA-MSC medium and at P4 in the OAC medium. Taken together, these data suggested that the transition of OAC to OAMSC was a gradual process comprising OAC dedifferentiation into NCSC-like MSC at P2 and acquiring the pro-inflammatory phenotype (SASP positive) at $\mathrm{P} 4$ in the OAC medium. In the OAMSC medium, the pro-inflammatory phenotype (SASP positive) was acquired at P3. Thus, high glucose and other energy sources in the OA-MSC medium accelerated the transition from the non-inflammatory OAC phenotype to the pro-inflammatory OAMSC phenotype associated with cell senescence.

\section{OAC, NCSC, and OA-MSC in Human OA Cartilage}

To further define the roles of OAC, NCSC, and OAMSC in cytokine and chemokine signaling, we analyzed the gene expression patterns of cytokines, chemokines, and their receptors (Figure 6). The RNAseq analysis showed that, while major cytokine and chemokines were synthesized by $\mathrm{OA}$ MSC (Figure 6A), OAC contained the highest expression of the receptors of cytokines and chemokines, while OA-MSC contained the lower levels and NCSC contained the lowest levels of cytokine receptor expression (Figure 6B). This suggested that, while OA-MSC was the source of inflammation, OAC was a major target cell of inflammation signaling in cartilage.

To determine the distribution of OAC and OA-MSC in human $\mathrm{OA}$ articular cartilage, we performed double immunohistochemical analysis using antibody against CD166, a marker of MSC (Figure 6A), and IL-1 $\beta$, a marker of SASP that was produced by OA-MSC (Figure 6A). Four types of cells were observed using these two markers: OAC (CD166-, IL-1 $\beta-$ ), OAC with cytokine on cell surface (CD166- IL$1 \beta+)$, NCSC (CD166+, IL-1 $\beta-$ ), and OA-MSC (CD166+, IL$1 \beta+)$ (Figure 6C). The CD166+ MSC cells in the superficial zone were negative for IL- $1 \beta$, indicating they were NCSC (Figure 6D, superficial; Figure 6E, NCSC). In contrast, many CD166+ MSC cells were positive for IL- $1 \beta$ in the middle and deep zones, indicating these were OA-MSC (Figure 6D, middle, deep; Figure 6E, OA-MSC). While OAC were abundantly distributed in the superficial and middle zones, their content was reduced in the deep zone (Figure 6D, deep; Figure 6E, OAC). Interestingly, many OACs in the middle zone were IL- $1 \beta+$ (Figure 6D, middle; Figure 6E, OAC cyto). This suggests that OAC is a recipient cell of cytokine signaling even though OAC does not synthesize IL-1 $\beta$ (Figure 6A).

\section{DISCUSSION}

Although OA was considered as a non-inflammatory disease, an ever-increasing body of evidence suggests that chronic degeneration of the joint is associated with persistent long-term low-grade inflammation in the joint (Jayasuriya et al., 2016). The source of inflammation in OA is unknown, although it has been shown to associate with high-fat diet, mechanical injury, and aging (Berenbaum, 2013). We have shown here that one of the sources of joint inflammation is OA-MSC within cartilage itself. OA-MSC synthesizes pro-inflammatory cytokines and chemokines that have been implicated in OA pathogenesis, including IL-1 $\beta$, IL-6, IL-8, CXCL1, 5, and 6 (Goldring, 2007). We demonstrated that the induction of such pro-inflammatory molecules occurs at both mRNA and protein levels through genome-wide RNAseq and protein array analysis, respectively. We have also shown that the induction of inflammation in OA cartilage occurs during the transition from normal cartilage stromal cell (NCSC) in the young to the OA-MSC in the old during aging.

In recent years, cell senescence has been shown to be closely associated with OA pathogenesis (Ming et al., 2018; 
A
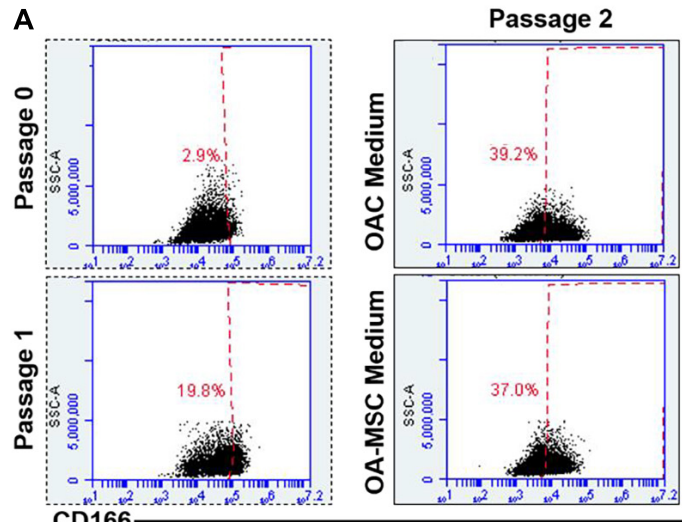
CD166

B
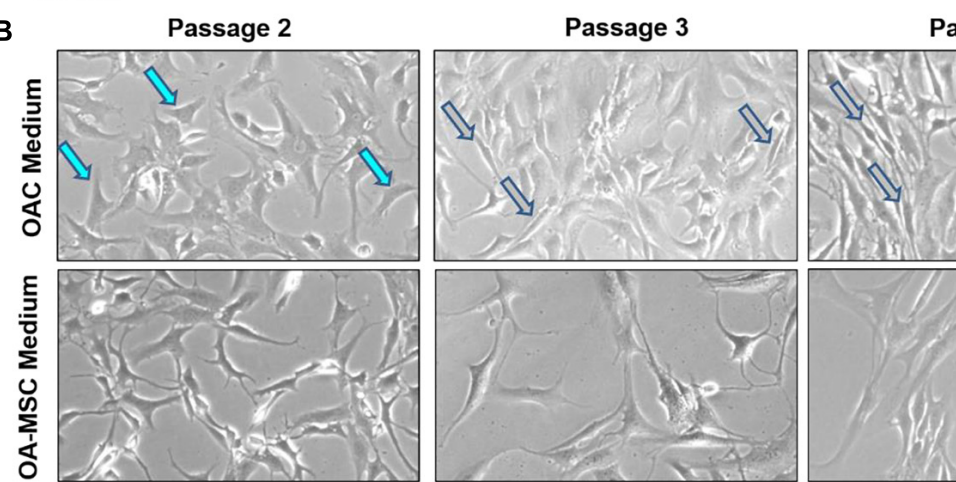

Passage 4
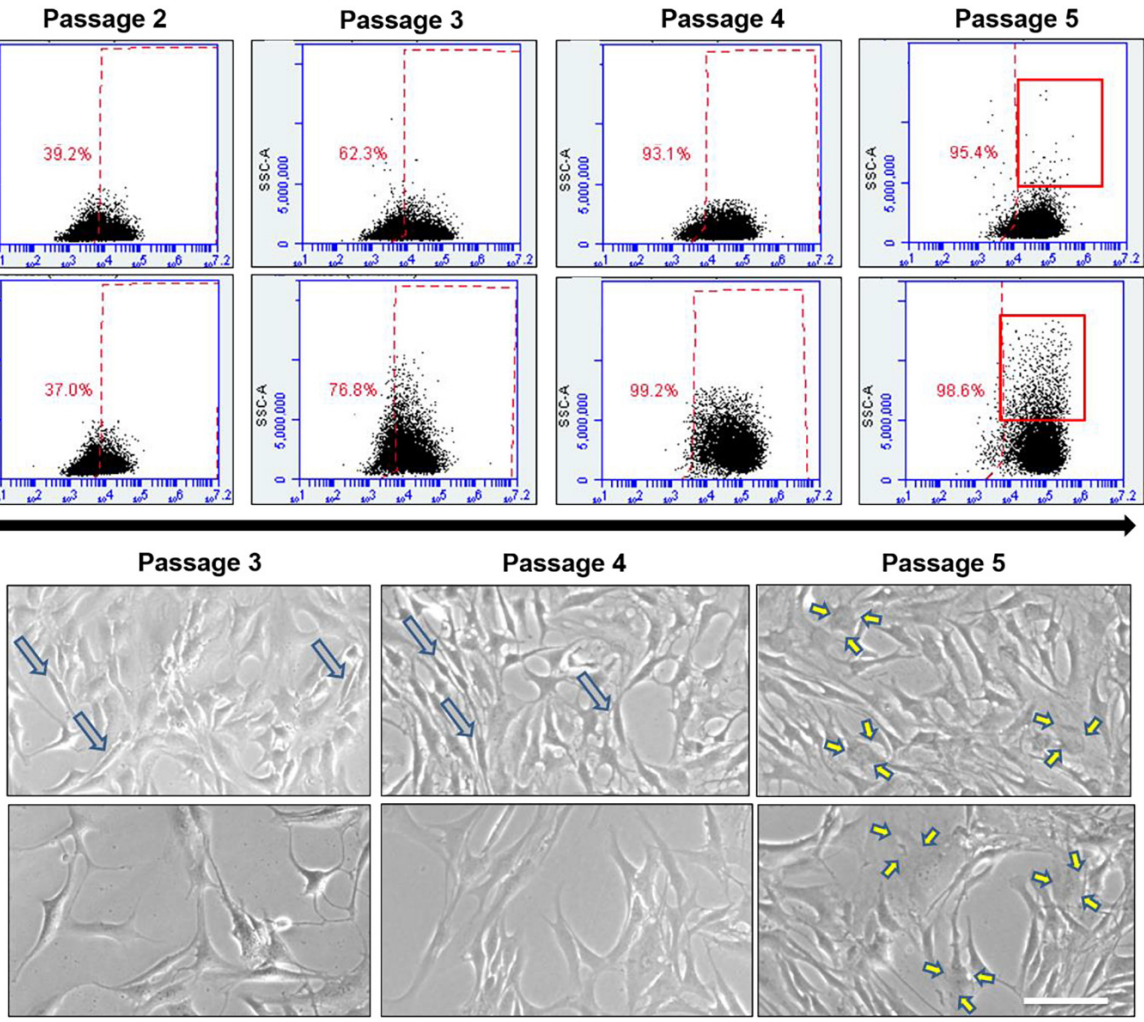

D
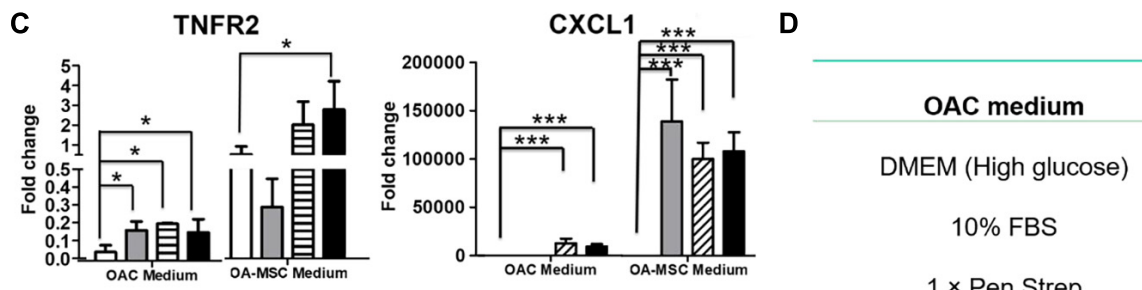

$10 \%$ FBS

$1 \times$ Pen Strep

Passage 5
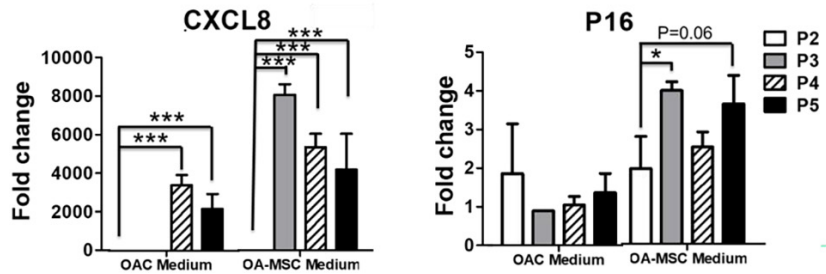

$0.5 \mathrm{mg} / \mathrm{mL}$ L-glucose

$$
1 \times \text { GlutaMAX }
$$

$0.1 \mathrm{mM}$ ascorbic acid

$1 \times$ sodium pyruvate

100 mM HEPES

FIGURE 5 | Serial cell culture passage of OAC induces dedifferentiation into NCSC-like cells and subsequent senescence into pro-inflammatory OA-MSC. (A) FACS analysis of CD166 positive cells during OA chondrocyte passaging in OA-MSC culture medium or OAC medium. The percentage of CD166 positive cells in each passage ( $x$-axis) was indicated in red. The increase of the number of large sized cells ( $y$-axis), a characteristic of senescent cells, was indicated by brackets in Passage 5. The data is representative of three experiments. (B) Cell morphology change of OA chondrocytes during cell culture passages in OAC medium and OA-MSC culture medium. OAC changed its morphology from cuboidal shape typical of chondrocytes (aquamarine arrows) in early passages (2 and 3 ) to spindle shape typical of fibroblasts (gray arrows) in mid passage (4) to large cells with processes typical of senescent cells (yellow arrows) in late passage (5). Bar $=200 \mu \mathrm{m}$. (C) Expression of aging related genes during OAC culture passages in OAC medium and OA-MSC medium. mRNA levels of TNFR2, CXCL1, CXCL8, P16 were quantified by real-time RT-PCR. The value of each sample group = mean \pm SD. $N=3$ for all groups. ${ }^{*} p$-value $<0.05 ;{ }^{* \star *} p$-value $<0.001$. (D) The components of OAC medium and OA-MSC medium.

Chu et al., 2020). Injection of senescent cells into the joint space led to joint degeneration (Xu et al., 2017). Conversely, local clearance of $\mathrm{p} 16^{I N K 4 a}$-positive senescent cells from the joint attenuated injury and aging induced OA (Jeon et al., 2017). Although the role of senescent cells in causing joint degeneration has been established, the molecular 


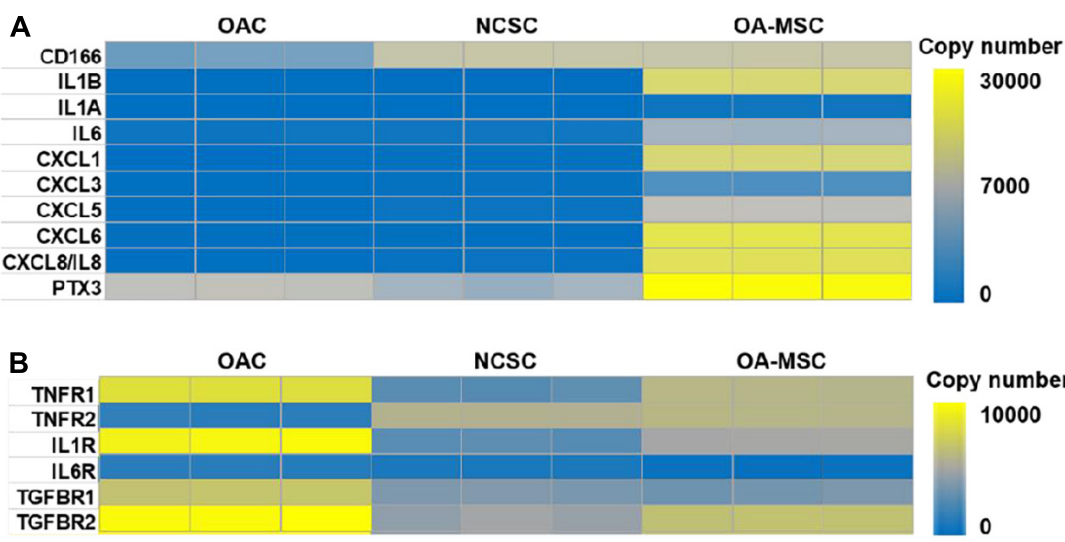

D

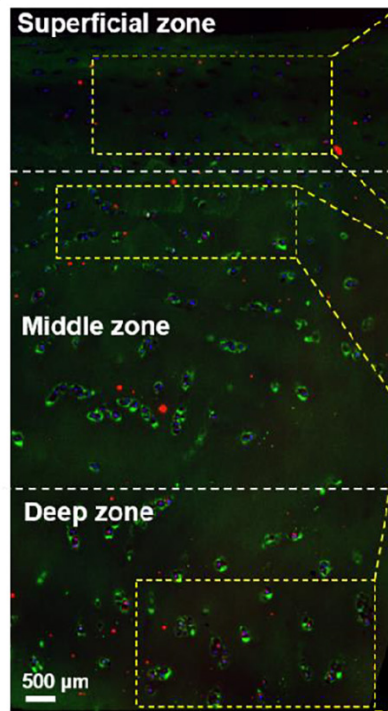

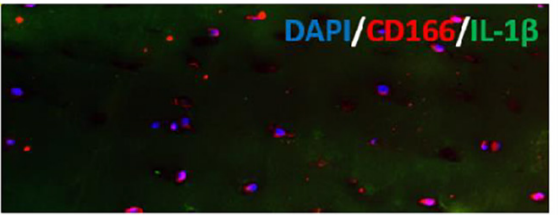

E
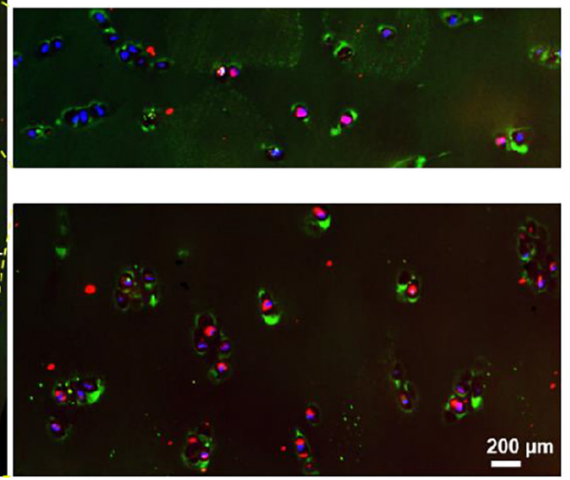

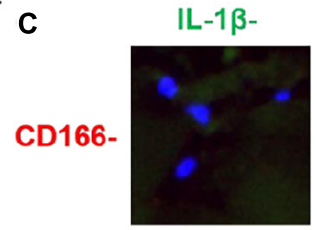

OAC

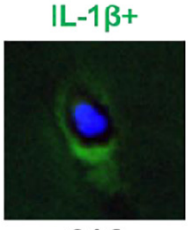

OAC (with cytokines)

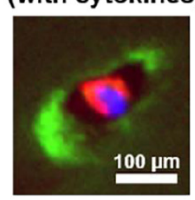

OA-MSC

NCSC

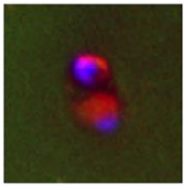

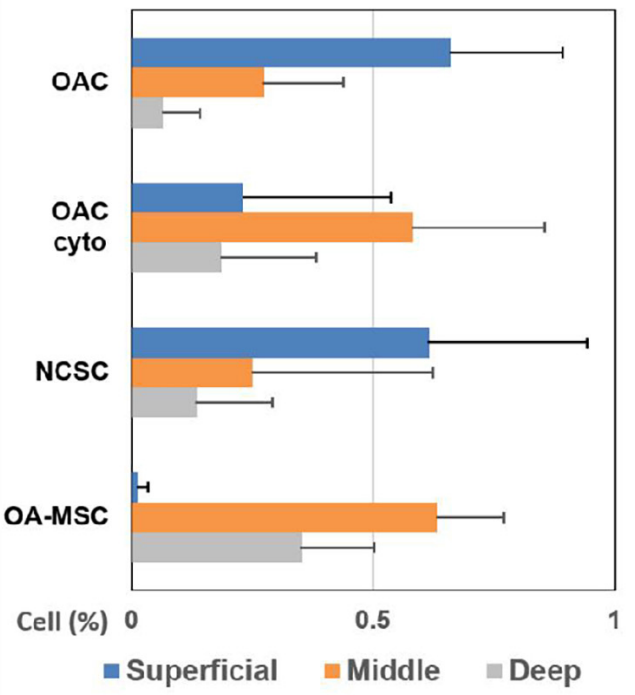

FIGURE 6 | Identification of OAC, NCSC-like cells, and OA-MSC in human OA articular cartilage. (A) Differential expression analysis of cytokine, chemokine, and related genes in OAC, NCSC, and OA-MSC. RNAseq analysis was performed with OAC, NCSC3, and OA-MSC2 samples ( $n=3$ ). Yellow color represents higher copy number up to 10,000 , while blue color represents lower copy number down to 0 . Gray color represents intermediate copy number. Note that while IL-1 $\beta$ was synthesized by OA-MSC, neither OAC nor NCSC synthesized IL-1 $\beta$. (B) Differential expression analysis of cytokine and chemokine receptors and related genes in OAC, NCSC, and OA-MSC. RNAseq analysis was performed with OAC, NCSC3, and OA-MSC2 samples ( $n=3)$. Yellow color represents higher copy number up to 30,000, while blue color represents lower copy number down to 0. Gray color represents intermediate copy number. (C) Immunofluorescence analysis of different types of cells in human OA cartilage. While chondrocytes are CD166-, mesenchymal stromal cells are CD166 ${ }^{+}$. OAC does not synthesize IL-1 $\beta$ but binds to IL-1 $\beta$ because it expresses IL-1R. OAC is CD166 $/ / \mathrm{LL}-1 \beta^{-}$; OAC binding to IL-1 $\beta$ (OAC with cytokine) is CD166 /IL-1 $\beta^{+}$, NCSC-like cell is CD166 ${ }^{+} / \mathrm{IL}_{-}-1 \beta^{-}$, and OA-MSC is

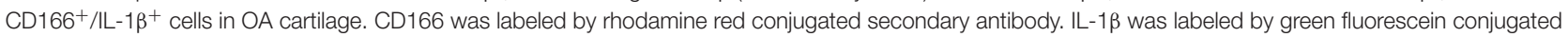
secondary antibody. The nucleus was stained with blue Hoechst dye. (D) The double immunofluorescence histochemical analysis of CD166 and IL-1 $\beta$ in different zones in human OA articular cartilage. CD166, a marker of MSC, was indicated by rhodamine-conjugated secondary antibody (red). IL-1 $\beta$, a marker of SASP was indicated by fluorescein-conjugated secondary antibody (green). The nucleus was stained with DAPI (blue). (E) The percentages of OAC, OAC (with cyto), NCSC-like cells, and OA-MSC in different zones of human OA articular cartilage $(n=3)$. Statistical analysis was presented in Supplementary Table 3.

mechanism by which a chondrocyte reaches senescence has not been well understood (Coryell et al., 2020). The expression levels of the cell senescence marker p16 INK4a and SASP were elevated in the serial passages of human chondrocyte culture in vitro and in aged human and mouse cartilage in vivo (Martin and Buckwalter, 2003). However, inactivation of $\mathrm{p}^{16^{I N K 4 a}}$ in chondrocytes of adult mice failed to attenuate joint degeneration during aging or injury (Diekman et al., 2018). This observation raised an important question whether senescent chondrocytes were involved in cartilage degeneration.

We have shown here for the first time that OA-MSC, but not OA chondrocytes (OAC), has elevated levels of $\mathrm{p} 16^{\text {INK4a }}$ and SASP. Therefore, OA-MSC, but not OAC, are the senescent cells that become a source of inflammation in the joint. Our study also provided a plausible molecular explanation to the observation that joint degeneration was not affected when the $\mathrm{p} 16^{\mathrm{INK} 4 a}$ gene was deleted in chondrocytes using the chondrocyte-specific 
Acan-Cre (Diekman et al., 2018). Since p16 is expressed at very low levels in the Acan-positive OAC (Figure 2C), targeting OAC for p16 knockout might not affect the real source of cell senescence in OA cartilage. Our data predict that joint degeneration would be attenuated if p16 were knocked out in OA-MSC, since it would abolish the source of SASP in OA cartilage. Although this prediction remains to be tested, OA-MSC should be considered as potential target cells of senolytics and anti-inflammation therapy for $\mathrm{OA}$ intervention in future studies.

Although OA-MSC is a source of SASP in cartilage, the receptors of cytokines and chemokines are expressed by both OAC and OA-MSC. In particular, IL-1R and TNFR1 are highly expressed by OAC. Although OAC does not synthesize IL$1 \beta$ mRNA, IL- $1 \beta$ protein is associated with OAC cell surface in human $\mathrm{OA}$ cartilage. It suggests that $\mathrm{OAC}$ is a recipient cell for pro-inflammatory signals secreted by OA-MSC. On the other hand, TNFR2 is highly expressed by OA-MSC but only at the moderate levels in OAC. Since TNFR1 and TNFR2 may activate different pathways (Wajant and Siegmund, 2019), TNF- $\alpha$ may activate different cellular responses in OAC and OA-MSC. Past studies have shown that IL-1 binding to its receptor on chondrocytes induce high levels of MMP-13, a major catabolic proteinase responsible for collagen degradation (Goldring, 2000a). We suggest an inflammation signaling axis in OA cartilage in which OA-MSC produces pro-inflammatory signals while $\mathrm{OAC}$ receives inflammation signals and activates catabolism and apoptosis (Figure 7A). Even though OA-MSC is much fewer in number than OAC in cartilage, it may act as seed senescent cells to release SASP affecting surrounding OAC. OAC may serve as executing cells that amplify the biological responses triggered by the inflammation signals from OA-MSC. The interplay between OA-MSC and OAC via NCSC alters the balance of anabolic matrix synthesis and catabolic ECM degradation (Figure 7C).

Bioinformatics analysis of RNAseq data supports the concept of the interplay between OA-MSC and OAC. It indicates that the main function of OA-MSC is twofold: (1) production of SASP including cytokines and growth factors; and (2) binding to chemokines and growth factors for signaling that results in cell fibrosis and senescence. It also indicates that the main function of OAC is twofold: (1) production of chondrogenic anabolic ECM for structural and regulatory functions, and (2) receptor signaling in response to the microenvironment, which in turn modifies the surrounding matrix and neighboring cells. While both OA-MSC and OAC exist in the same aged OA cartilage, OA-MSC is senescent and OAC is not; OA-MSC is fibrotic and pro-inflammatory and OAC is chondrogenic and does not produce SASP. Thus, our data suggests that not all cells in an aged tissue are senescent. It is a specific type of cell, in this case senescent OA-MSC, which are responsible for the fibrotic and pro-inflammatory phenotype of an aged tissue. Since OA-MSC is much fewer in number than OAC, it suggests that cell senescence could be initiated in a few seed cells that become the foci of cell senescence in a degenerative tissue during aging.

Our study suggests that cell type transitions may occur during cell aging and senescence process (Figure 7B). They result in the interaction of different types of cells, which lead to cell death and tissue degeneration (Figure 7A). This process in termed "Senescence Associated Cell Transition and Interaction" (SACTAI). Testing the SACTAI concept will help our understanding of the cellular processes that lead to cell senescence, inflammation, and degradation. The GSEA bioinformatics analysis indicated that SACT is similar to epithelial-mesenchymal transition (EMT), although it would be more appropriate to term it chondro-mesenchymal transition (CMT) during cartilage aging and senescence. There are many similarities between SACT and EMT. First, like EMT, CMT involves attenuation of tissue specific chondrogenic gene expression, chondrocyte morphology and matrix adhesion, and a gain of migratory and invasive properties including secretion of pro-inflammatory cytokine, chemokine, and MMPs. Second, both CMT and EMT involve cell phenotype conversion from a specialized quiescent somatic cell to a mesenchymal stem cell capable of self-replicating and subsequent differentiation into different cell lineages. Third, EMT has been shown to occur not only during development and organogenesis but also in the physiological and pathological processes during adulthood including wound healing, tissue fibrosis, and cancer progression (Georgakopoulos-Soares et al., 2020). Since cell senescence has been shown to be involved in these events, SACTAI will likely play an important role not only during development but also in adult tissue homeostasis and aging.

Our study also suggests SACTAI may be induced by repeatedly activation of cell replication. Replicative senescence of MSC during joint aging is supported by the literature on MSCs harvested beneath the articular cartilage (Vozzi et al., 2016; Dicarlo et al., 2018), and MSCs from synovium (Dicarlo et al., 2019). In the original discovery of replicative cell senescence Hayflick and Moorhead (1961) isolated human fibroblasts from fetal lung and cultured to reach confluence. Cell proliferation was activated repeatedly in a series of cell passages in culture. After about 50 culture sub-cultivations, cells reached senescence before death. Subsequently it was discovered that senescent cells synthesized pro-inflammatory proteins termed SASPs (Coppé et al., 2011). In this study, we performed similar serial cell culture passages of aged osteoarthritic human chondrocytes to repeatedly activate cell proliferation. We found that it took as few as five cell passages to reach cell senescence. Chondrocyte replication in serial culture passages induced cell dedifferentiation and subsequent senescence. During dedifferentiation of primary chondrocytes into fibroblastic MSCs, the chondrogenic phenotype of OAC was suppressed by transcriptional repression, resulting in NCSC-like MSCs. We also observed such NCSC-like MSCs (CD166+; IL-1 $\beta-$ ) in human OA cartilage (Figure 6). Thus these transitional MSCs may exist in vivo. Such OAC-derived NCSC-like cells were also observed by other laboratories previously (Oda et al., 2016). Low glucose concentrations were shown to be critical for maintaining the regenerative capacities of these NCSC-like cells (Han et al., 2004). We show here that high glucose leads to accelerations of MSC senescence to pro-inflammatory OA-MSC (Figure 7B).

Our RNAseq analysis indicates that, during MSC senescence, the inflammatory and fibrotic phenotypes of OA-MSC were 
A

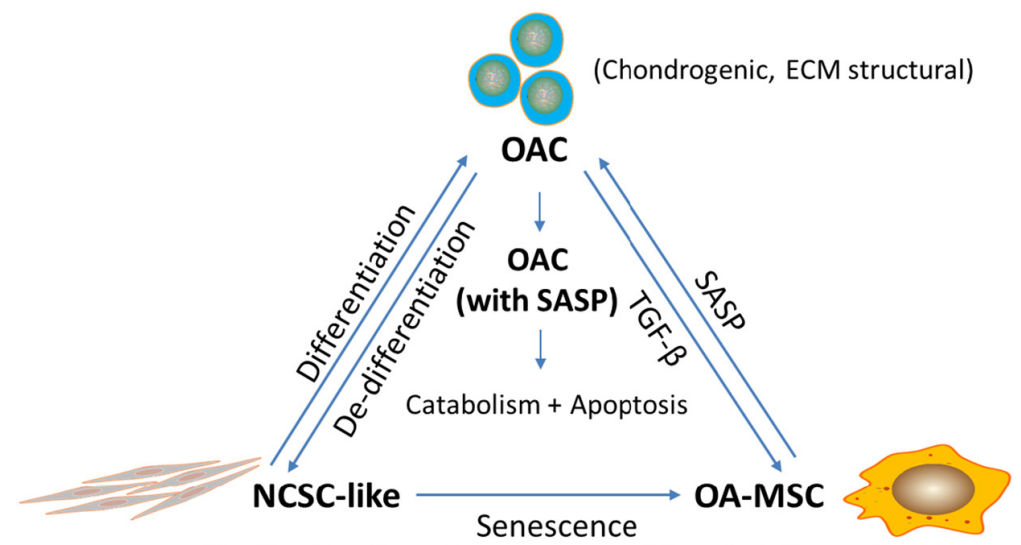

(Proliferative, Repair)

(Fibrosis, Inflammation)

B
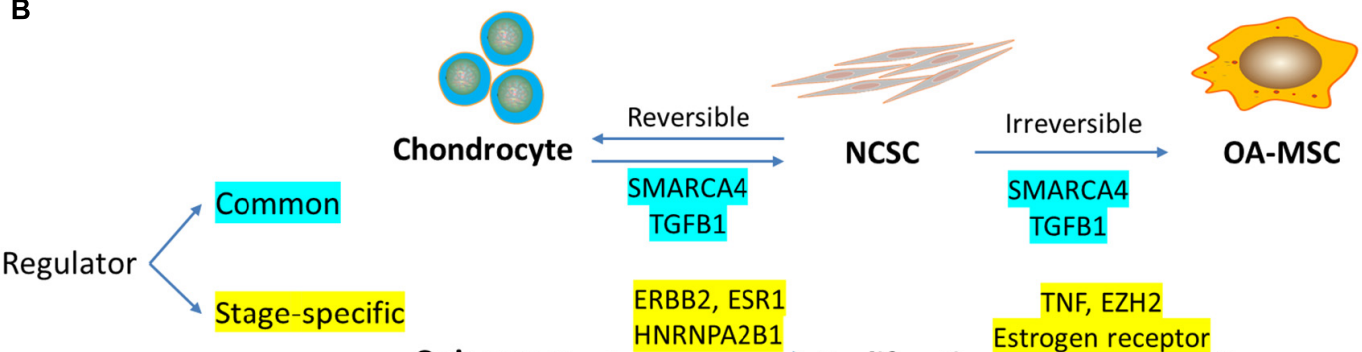

Quiescence

ERBB2, ESR1

TNF, EZH2

HNRNPA2B1

Low Glucose

Proliferation

Estrogen receptor

$\overrightarrow{\text { High Glucose }}$

Senescence

C

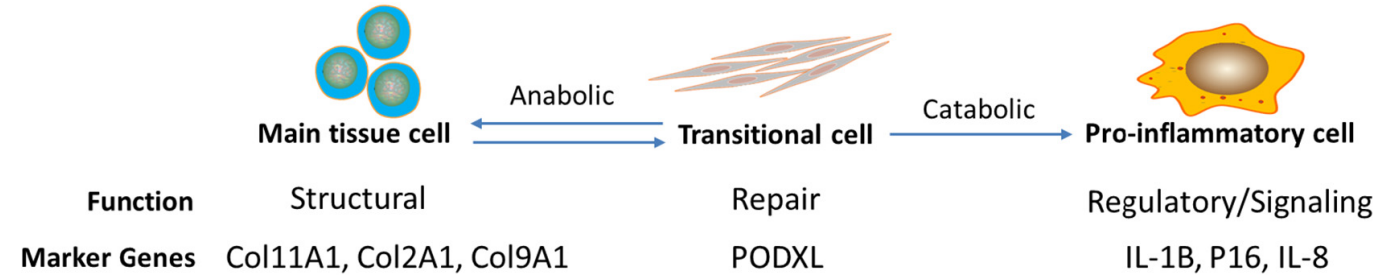

FIGURE 7 | RNA sequencing analysis indicates the relationship among chondrocytes, mesenchymal stromal cells (MSC), and senescent MSC in human articular cartilage during aging. (A) Working hypothesis of the relationship among OAC, NCSC-like cells, and OA-MSC in aged human OA cartilage. Cartilage defect due to aging, stress, or injury induces proliferation and dedifferentiation of OAC, which results in NCSC-like cells for tissue repair. Repeated activation of NCSC-like cell replication in aged cartilage induces replicative senescence, which results in senescent OA-MSC. OA-MSC synthesizes pro-inflammatory SASP including IL-1b, IL-6, CXCL1, 5, 6, and 8. Upon binding its receptors on OAC, SASP activates cytokine-induced catabolism and apoptosis of OAC, thereby contributing to joint degeneration and OA pathogenesis. (B) Regulators of Senescence Associated Cell Transition (SACT) during cartilage aging. RNAseq analysis identified common and stage-specific regulators of cell transitions during chondrocyte aging process. The working hypothesis is that SACT comprises two cell transitions during aging. The first transition converts quiescent normal adult articular chondrocytes into proliferative and de-differentiated NCSC. The second transition converts proliferative NCSC into senescent and pro-inflammatory OA-MSC. The common regulators governing both transitions include SMARCA4 and TGFB1. The stage-specific regulators for the first transition include ERBB2, ESR1, and HNRNPA2B1. The specific regulators for the second transition include TNF, EZH2, and estrogen receptor. While the transition of chondrocytes to NCSC or vice versa is reversible, the transition of NCSC to OA-MSC may be irreversible. While high glucose drives MSC senescence and inflammation, low glucose may maintain the NCSC/chondrocyte balance for tissue repair (Jiang et al., 2016). (C) Proposed function and marker genes for OAC, NCSC, and OA-MSC in human articular cartilage. The working hypothesis is that chondrocytes serve a structural function as the main tissue resident cells in cartilage; NCSC serve as a transitional cell for tissue repair and wound healing in response to stress, injury, or aging; and OA-MSC serve as a regulatory, signaling cell during injury, and aging process. The marker genes for chondrocytes include chondrogenic ECM COL11A1, COL2A1, and COL9A1; NCSC marker gene includes MSC marker PODXL; and OA-MSC marker genes include cell senescence and SASP markers IL1B, P16, and IL8.

acquired by transcriptional activation. Serial passages of primary OAC not only decreased the percentage of chondrocytes but also increased the percentage of MSC, which ultimately resulted in senescent OA-MSC. A shortcoming of this study is that we did not determine the percentage of OA-MSC derived from cell type transition or proliferation. Such analysis requires performing in vivo cell lineage tracing studies in the future. Through immunohistochemistry analysis, we observed multiple cell types in human OA cartilage including chondrocytes (CD166-) and MSCs (CD166+) in both SASP positive and negative categories. 
Existence of these multiple types of cells in human OA cartilage not only suggests their interactions with each other spatially (Figure 7A), but also potential cell type transitions temporally (Figure 7B). Recent single-cell RNAseq analysis also supports the concept of multiple cell types including chondrocytes, MSC (chondroprogenitor cells), and derived cell types in human OA cartilage (Ji et al., 2019). Importantly, our study here identified not only the senescent cell type and its markers in human OA cartilage (Figure 7C), but also the critical regulators driving chondrocyte dedifferentiation and MSC senescence (Figure 7B). The pro-inflammatory cytokines and chemokines secreted by OA-MSC may play a critical role in regulating OA pathogenesis in the joint during aging.

\section{MATERIALS AND METHODS}

\section{Human Tissue}

Human articular cartilage tissues were collected from OA patients (Male and Female, Age: 56-85 years old) who underwent total knee replacement (OA cartilage) and young adult cancer patient (Female, 18 years old) who underwent leg amputation (normal cartilage). The use of discarded human cartilage tissues were approved by the Institutional Review Board (IRB) of Rhode Island Hospital.

\section{Cell Isolation and Cultivation}

The OA-MSCs and OA were isolated from the normal looking tissue area of the full thickness OA articular cartilage. Cartilage samples were rinsed three times with PBS plus $1 \%$ penicillinstreptomycin and then cut into small pieces. The cartilage fragments were digested by $2.0 \mathrm{mg} / \mathrm{mL}$ Pronase (Roche, Indianapolis, IN, United States) for $30 \mathrm{~min}$ at $37^{\circ} \mathrm{C}$ with shaking water bath. Then cartilage fragments were rinsed with PBS three times and treated with $1.0 \mathrm{mg} / \mathrm{mL}$ Collagenase I (SigmaAldrich, St. Louis, MO, United States) in DMEM overnight in $37^{\circ} \mathrm{C}$ shaking water bath. Cells with medium were strained through a nylon cell strainer $(100 \mu \mathrm{m})(\mathrm{BD}$, Franklin Lakes, NJ, United States) and rinsed with DMEM three times. Cells were plated in fibronectin $(10 \mu \mathrm{g} / \mathrm{mL}$ in PBS) coated dishes for $20 \mathrm{~min}$ at $37^{\circ} \mathrm{C}$ in cell incubator. After $20 \mathrm{~min}$, the adherent cells were primary OA-MSC while non-adherent cells were collected as OA chondrocytes. The OA-MSCs were cultured in OA-MSC medium: DMEM with $10 \%$ FBS, $1 \%$ penicillinstreptomycin, $100 \mathrm{mM}$ HEPES, $1 \%$ GlutaMax, $0.1 \mathrm{mM}$ ascorbic acid, $0.1 \mathrm{mM}$ sodium pyruvate, and $2.7 \mu \mathrm{M}$ L-glucose. The OAC were cultured in OAC medium: DMEM with $10 \% \mathrm{FBS}$, $1 \%$ penicillin-streptomycin, $100 \mathrm{mM}$ HEPES, unless noted otherwise.

Establishment and characterization of OA-MSC cell lines and normal cartilage stromal cell (NCSC) lines were described previously (Jayasuriya et al., 2018, 2019). The OA-MSC and NCSC cell lines were found to retain the gene expression pattern and multi-lineage differentiation potentials of primary cell counterpart, respectively (Jayasuriya et al., 2018, 2019). The cartilage-derived OA-MSC and NCSC cell lines retain the MSC marker expression pattern and multi-lineage differentiation potentials as the primary MSC (Jayasuriya et al., 2018, 2019; Hu et al., 2019; Liu et al., 2020). The primary OAC and OAMSC were from the same cartilage tissue thus eliminating the systemic differences between different tissues or patients. While OA-MSC was from old OA cartilage, NCSC was from young normal cartilage. Thus, the difference of OA-MSC and NCSC represents cell senescence changes during aging.

\section{Human Cytokine Protein Array}

We performed protein expression analysis of OA-MSC, NCSC, OAC, and primary OA-MSC using a Human XL Cytokine Array Kit (R\&D system, Minneapolis, MN, United States). All the procedures and data analysis were performed following the manufacturer instructions. Total protein was extracted from $1 \times 10^{7}$ cells using Lysis Buffer 17 (R\&D system, Minneapolis, $\mathrm{MN}$, United States) supplemented with $10 \mu \mathrm{g} / \mathrm{mL}$ Aprotinin (Tocris Bioscience, Minneapolis, MN, United States), $10 \mu \mathrm{g} / \mathrm{mL}$ Leupeptin (Tocris Bioscience, Minneapolis, MN, United States), and $10 \mu \mathrm{g} / \mathrm{mL}$ Pepstatin (Tocris Bioscience, Minneapolis, MN, United States). Each sample containing $200 \mu$ g protein in $1.5 \mathrm{~mL}$ Array Buffer 6 was run on each array (membrane) under incubation at $4^{\circ} \mathrm{C}$ overnight on a shaker. Then the membrane was washed three times with Wash Buffer for $10 \mathrm{~min}$ on a shaker. Twenty microliters of Detection Antibody Cocktail in $1.5 \mathrm{~mL}$ Array Buffer $4 / 6$ was added to each membrane for $1 \mathrm{~h}$ incubation on a shaker. $1 \times$ Streptavidin-HRP was added for $30 \mathrm{~min}$ after three times washing with Wash Buffer. The membrane was incubated with $1.0 \mathrm{~mL}$ Chemi Reagent Mix for $1 \mathrm{~min}$ with plastic sheet protector. The liquid was then squeezed out by paper towels. The membranes were exposed by ChemiDoc MP (BioRad, Hercules, CA, United States) to generate the profile of spot pixel density image. The density signals of each spot were quantified by Image Pro Plus. The relative differences of sample signals were compared based on the standardized signal unit.

\section{RNA Purification and Sequencing}

Total RNA was purified from cultured cells using Qiagen RNAeasy kit. RNA sequencing was performed by Genewiz, Inc. Sequence reads were trimmed to remove possible adapter sequences and nucleotides with poor quality using Trimmomatic v.0.36. The reads were then mapped to the Homo sapiens GRCh38 reference genome available on ENSEMBL using the STAR aligner. The RNA-seq aligner is executed using a splice aligner which detects splice junctions and incorporating them to help align the entire read sequences. BAM files were generated as a result of this step. Unique exon hit counts were calculated using Feature counts from the Subread package. The top 30 significantly changed genes (fold change $>2$ ) were selected by the lowest $p$-values to create Bi-Clustering Heat Map.

\section{Volcano Plot}

All the genes are plotted for the volcano plot and each point represents one gene. The $x$-axis represents Log2FoldChange while the $y$-axis represents $\log 10$ of $p$-value. An adjusted $p$-value $<0.05$ and a Log2FoldChange $>1$ are indicated in 
OA-MSC, OAC, and NCSC by yellow dots, blue dots, and gray dots, respectively.

\section{Gene Ontology (GO) Analysis}

PANTHER System was used for the GO analysis (Mi et al., 2019). The genes with $p$-value $<0.05$ and fold change $>2$ were selected for the GO analysis. The "GO molecular function complete" mode was selected to generate a list of related gene numbers for each classification. The top 20 significant classifications with gene numbers in each comparison were selected to form a bar chart.

\section{IPA Pathway Analysis}

Qiagen IPA system was used for analysis. All the analysis was performed based on base mean $>300, p$-value $<0.05$, fold change $>2$. The top analysis and pathway analysis were performed in OA-MSC vs. OAC, OA-MSC vs. NCSC, and NCSC vs. OAC. The top 20 activated or inhibited pathways were selected into a bar graph. The red, green, and blue bars represented activated pathway in OA-MSC, OAC, and NCSC, respectively. The deeper in color means higher activation. The $x$-axis represents for $\log 10$ of $p$-value.

\section{Gene Set Enrichment Analysis (GSEA)}

The RNAseq data was analyzed by pre-rank GSEA v3 (GSEA $\mathrm{REF}^{1}$ ) using the MsigDB v6.1 gene sets. ${ }^{2}$ NES $>1.5$ and FDR $<0.05$ were considered significant.

\section{Real-Time PCR}

Total messenger RNA was isolated from cells using a Qiagen RNeasy Kit (Qiagen, Hilden, Germany) according to instructions. Each sample of $500 \mathrm{ng}$ RNA was reverse transcribed using the miScriptIIRT Kit (Qiagen, Hilden, Germany). Real-time PCR was performed with the SYBR Green PCR Master mix (Qiagen, Hilden, Germany) using the Bio-Rad CFX96 real-time PCR detection system (Bio-Rad, CA, United States). The forward and reverse primer sequences were listed in Supplementary Table 2. Ribosomal RNA $18 \mathrm{~S}$ was used as the reference gene for normalization. Relative gene expression level was determined by the $2^{-\Delta \Delta C t}$ method.

\section{Flow Cytometry}

Pre-conjugated antibodies CD166-FITC and mouse-IgG-FITC were purchased from Miltenyi Biotec (Bergisch Gladbach, Germany). Cells were washed twice with PBS and detached using TrypLE Express (Life Technologies, Grand Island, NY, United States). Cells were re-suspended with DMEM plus 10\% FBS. Cells were then centrifuged of $300 \times g$ and re-suspended with PBS twice. Next, $1.0 \times 10^{6}$ cells were re-suspended in $100 \mu \mathrm{L}$ Flow buffer (PBS ( $\mathrm{pH} 7.2$ ) with $0.5 \%$ bovine serum albumin and $2 \mathrm{mM}$ EDTA). Ten microliters pre-conjugated antibody or negative control was added to each resuspension, mixed, and incubated for $10 \mathrm{~min}$ at $4^{\circ} \mathrm{C}$ in the dark. Cells were rinsed three times with PBS and re-suspended in $500 \mathrm{~mL}$ Flow buffer. Accuri

\footnotetext{
${ }^{1}$ https://pubmed.ncbi.nlm.nih.gov/16199517/

${ }^{2}$ https://www.ncbi.nlm.nih.gov/pmc/articles/PMC4707969/
}

C6 Flow Cytometer (BD Biosciences, San Jose, CA, United States) was used for flow cytometry analysis.

\section{Immunohistochemistry}

Human OA articular cartilage of full thickness $(n=3)$ was processed and sectioned as previously described (Liu et al., 2020). The sections were treated by $3 \%$ hydrogen peroxide in methanol for $30 \mathrm{~min}$, and by $100 \mathrm{mg} / \mathrm{mL}$ hyaluronidase for 15 min. After blocking non-specific binding with 3\% bovine serum albumin (BSA) in PBS, immunohistochemistry was performed with primary antibodies (mouse anti-CD166 Abcam, MA, United States, catalog number: ab233750), 1:100; rabbit anti-IL-1 $\beta$ (Santa Cruz Biotechnology, TX, United States, catalog number: sc-7884) at $4^{\circ} \mathrm{C}$ overnight. Sections were stained for $30 \mathrm{~min}$ with a red fluorescently labeled anti-mouse secondary antibody and/or a green fluorescently labeled antirabbit secondary antibody (Invitrogen, MA, United States). The VECTASHIELD Mounting Medium with DAPI was used for cell nuclear staining and section storage. The section was Images were acquired at $4 \times$ and $20 \times$ magnification using a Nikon Eclipse $90 \mathrm{i}$ Digital Imaging System.

\section{Western Blot Analysis}

Cells were washed, lysed, and processed as previously described (Liu et al., 2020). Equal amount of proteins for each sample were separated by $10 \%$ SDS-polyacrylamide gel and then transferred to Nitrocellulose (NC) membrane for $70 \mathrm{~min}$ at $100 \mathrm{~V}$. The membrane was blocked with $5 \%$ bovine serum albumin (BSA) in Tris-buffered saline-Tween 20 (0.1\%) (TBST) for $1 \mathrm{hr}$ at room temperature. Rabbit anti-TNFR2 antibody (Abcam, MA, United States, ab109322) were used at 1:1000 dilution at $4^{\circ} \mathrm{C}$ overnight. $\beta$-Actin was detected using anti-rabbit antibody (Abcam, MA, United States, ab179467) as reference protein. Membrane was rinsed with TBS-T for $10 \mathrm{~min}$, five times and incubated with 1:5000 goat anti-rabbit-Alexa Fluor 680 or donkey anti-mouse-Alexa Fluor 680 (Molecular Probes, MA, United States) for $1 \mathrm{~h}$ at room temperature. The blots were scanned using an Odyssey fluorescence scanner (LI-COR Biosciences, NE, United States).

\section{Statistics}

Unpaired student's $t$-test was used for gene expression analysis in each two groups, which represent mean values \pm SD (error bars). $p$-Value $<0.05$ were considered statistically significant.

\section{DATA AVAILABILITY STATEMENT}

The data presented in the study are deposited in the NCBI GEO repository, accession number: GSE176199.

\section{ETHICS STATEMENT}

The studies involving human participants were reviewed and approved by Rhode Island Hospital IRB Committee. The 
patients/participants provided their written informed consent to participate in this study.

\section{AUTHOR CONTRIBUTIONS}

WL contributed to design of the study, acquisition, analysis and interpretation of the data, drafting the manuscript and revising the content, and approval of the final version. $\mathrm{AB}$ contributed to analysis and interpretation of the data, revising manuscript, and approval of the final version. MF contributed to acquisition, analysis and interpretation of the data, revising manuscript, and approval of the final version. YL contributed to acquisition, analysis and interpretation of the data, revising manuscript, and approval of the final version. JD contributed to acquisition of the data, revising manuscript, and approval of the final version. CJ contributed to providing experimental samples, revising manuscript, and approval of the final version. QC contributed to conception and design of the study, analysis and interpretation of the data, drafting the manuscript and revising the content, and approval of the final

\section{REFERENCES}

Alsalameh, S., Amin, R., Gemba, T., and Lotz, M. (2004). Identification of mesenchymal progenitor cells in normal and osteoarthritic human articular cartilage. Arthritis Rheum. 50, 1522-1532. doi: 10.1002/art.20269

Berenbaum, F. (2013). Osteoarthritis as an inflammatory disease (osteoarthritis is not osteoarthrosis!). Osteoarthritis Cartilage 21, 16-21. doi: 10.1016/j.joca.2012. 11.012

Borzì, R., Mazzetti, I., Cattini, L., Uguccioni, M., and Facchini, A. (2000). Human chondrocytes express functional chemokine receptors and release matrixdegrading enzymes in response to C-X-C and C-C chemokines. Arthritis Rheumatol 43, 1734-1741.

Chu, X., Wen, J., and Raju, R. P. (2020). Rapid senescence like response after acute injury. Aging Cell 2020:e13201. doi: 10.1111/acel.13201

Coppe, J., Desprez, P. Y., Krtolica, A., and Campisi, J. (2010). The senescenceassociated secretory phenotype: the dark side of tumor suppression. Annu. Rev. Pathol. 5, 99-118. doi: 10.1146/annurev-pathol-121808-102144

Coppé, J.-P., Rodier, F., Patil, C. K., Freund, A., Desprez, P. Y., and Campisi, J. (2011). Tumor suppressor and aging biomarker p16INK4aInduces cellular senescence without the associated inflammatory secretory phenotype. J. Biol. Chem. 286, 36396-36403. doi: 10.1074/jbc.m111.257071

Coryell, P. R., Loeser, R. F., and Diekman, B. O. (2020). Mechanisms and therapeutic implications of cellular senescence in osteoarthritis. Nat. Rev. Rheumatol. 17, 47-57. doi: 10.1038/s41584-020-00533-7

Dicarlo, M., Teti, G., Cerqueni, G., Iezzi, I., Gigante, A., Falconi, M., et al. (2019). Synovium-derived stromal cell-induced osteoclastogenesis: a potential osteoarthritis trigger. Clin. Sci. 133, 1813-1824. doi: 10.1042/CS20190169

Dicarlo, M., Teti, G., Iezzi, I., Cerqueni, G., Manzotti, S., Falconi, M., et al. (2018). Detecting senescent fate in mesenchymal stem cells: a combined cytofluorimetric and ultrastructural approach. Biogerontology 19, 401-414. doi: 10.1007/s10522-018-9766-4

Diekman, B. O., Sessions, G. A., Collins, J. A., Knecht, A. K., Strum, S. L., Mitin, N. K., et al. (2018). Expression of p16INK4ais a biomarker of chondrocyte aging but does not cause osteoarthritis. Aging Cell 17:e12771. doi: 10.1111/acel.12771

Dowthwaite, G. P., Bishop, J. C., Redman, S. N., Khan, I. M., Rooney, P., Evans, D. J., et al. (2004). The surface of articular cartilage contains a progenitor cell population. J. Cell Sci. 117, 889-897. doi: 10.1242/jcs.00912

Edith, C., Céline, D., Federica, C., Olivier, M., Sophie, N., Zelda, P., et al. (2019). Chondrocyte dedifferentiation and osteoarthritis (OA). Biochem. Pharmacol. 165, 49-65. doi: 10.1016/j.bcp.2019.02.036 version. All authors contributed to the article and approved the submitted version.

\section{FUNDING}

We gratefully acknowledge financial support from $\mathrm{NIH}$ (P30GM122732 and 1R61AR076807-01) to QC.

\section{ACKNOWLEDGMENTS}

We thank John Froehlich and Richard Terek for providing discarded human cartilage samples from surgery.

\section{SUPPLEMENTARY MATERIAL}

The Supplementary Material for this article can be found online at: https://www.frontiersin.org/articles/10.3389/fcell.2021. 725071/full\#supplementary-material

Fellows, C. R., Williams, R., Davies, I. R., Gohil, K., Baird, D. M., Fairclough, J., et al. (2017). Characterisation of a divergent progenitor cell sub-populations in human osteoarthritic cartilage: the role of telomere erosion and replicative senescence. Sci. Rep. 7:41421. doi: 10.1038/srep41421

Georgakopoulos-Soares, I., Chartoumpekis, D. V., Kyriazopoulou, V., and Zaravinos, A. (2020). EMT factors and metabolic pathways in cancer. Front. Oncol. 10:499. doi: 10.3389/fonc.2020.00499

Goldring, M. B. (2000a). Osteoarthritis and cartilage: the role of cytokines. Curr. Rheumatol. Rep. 2, 459-465. doi: 10.1007/s11926-000-0021-y

Goldring, M. B. (2000b). The role of the chondrocyte in osteoarthritis. Arthritis Rheum. 43, 1916-1926. doi: 10.1002/1529-0131(200009)43:93.0.CO;2-I

Goldring, M. B. (2007). Cytokines, growth factors, and bone-derived factors in cartilage. Bone Osteoarthritis 4, 41-63. doi: 10.1007/978-1-84628-701-5_3

Goldring, M. B. (2012). Chondrogenesis, chondrocyte differentiation, and articular cartilage metabolism in health and osteoarthritis. Ther. Adv. Musculoskel. 4, 269-285. doi: 10.1177/1759720X12448454

Han, Y. S., Bang, O. S., Jin, E. J., Park, J. H., Sonn, J. K., and Kang, S. S. (2004). High dose of glucose promotes chondrogenesis via PKC $\alpha$ and MAPK signaling pathways in chick mesenchymal cells. Cell Tissue Res. 318, 571-578. doi: 10. 1007/s00441-004-0993-4

Hayflick, L., and Moorhead, P. S. (1961). The serial cultivation of human diploid cell strains. Exp. Cell Res. 25, 585-621. doi: 10.1016/0014-4827(61)90 $192-6$

Hu, N., Gao, Y., Jayasuriya, C. T., Liu, W., Du, H., Ding, J., et al. (2019). Chondrogenic induction of human osteoarthritic cartilage-derived mesenchymal stem cells activates mineralization and hypertrophic and osteogenic gene expression through a mechanomiR. Arthritis Res. Ther. 21:167. doi: 10.1186/s13075-019-1949-0

Jayasuriya, C. T., Chen, Y., Liu, W., and Qian, C. (2016). The influence of tissue microenvironment on stem cell-based cartilage repair. Ann. Ny. Acad. Sci. 1383, 23-31. doi: 10.1111/nyas.13170

Jayasuriya, C. T., Hu, N., Li, J., Lemme, N., Terek, R., Ehrlich, M. G., et al. (2018). Molecular characterization of mesenchymal stem cells in human osteoarthritis cartilage reveals contribution to the OA phenotype. Sci. Rep. UK 8:7044. doi: 10.1038/s41598-018-25395-8

Jayasuriya, C. T., Twomey-Kozak, J., Newberry, J., Desai, S., Feltman, P., Franco, J. R., et al. (2019). Human cartilage-derived progenitors resist terminal differentiation and require CXCR4 activation to successfully bridge meniscus tissue tears. Stem Cells 37, 102-114. doi: 10.1002/stem. 2923 
Jeon, O. H., Kim, C., Laberge, R. M., Demaria, M., Rathod, S., Vasserot, A. P., et al. (2017). Local clearance of senescent cells attenuates the development of posttraumatic osteoarthritis and creates a pro-regenerative environment. Nat. Med. 23, 775-781. doi: 10.1038/nm.4324

Ji, Q., Zheng, Y., Zhang, G., Hu, Y., Fan, X., Hou, Y., et al. (2019). Single-cell RNAseq analysis reveals the progression of human osteoarthritis. Ann. Rheum. Dis. 78, 100-110. doi: 10.1136/annrheumdis-2017-212863

Jiang, Y., Cai, Y., Zhang, W., Yin, Z., Hu, C., Tong, T., et al. (2016). Human cartilage-derived progenitor cells from committed chondrocytes for efficient cartilage repair and regeneration. Stem Cells Transl. Med. 5, 733-744. doi: 10.5966/sctm.2015-0192

Liu, W., Feng, M., Jayasuriya, C. T., Peng, H., Zhang, L., Guan, Y., et al. (2020). Human osteoarthritis cartilage-derived stromal cells activate joint degeneration through TGF-beta lateral signaling. FASEB J. 34, 16552-16566. doi: 10.1096/fj.202001448R

Martin, J. A., and Buckwalter, J. A. (2003). The role of chondrocyte senescence in the pathogenesis of osteoarthritis and in limiting cartilage repair. J. Bone. Joint. Surg. Am. 85(A Suppl. 2), 106-110. doi: 10.1054/arth.2003.5 0034

Mendler, M., Eich-Bender, S. G., Vaughan, L., Winterhalter, K. H., and Bruckner, P. (1989). Cartilage contains mixed fibrils of collagen types II, IX, and XI. J. Cell Biol. 108, 191-197. doi: 10.1083/jcb.108.1.191

Mi, H., Muruganujan, A., Huang, X., Ebert, D., Mills, C., Guo, X., et al. (2019). Protocol Update for large-scale genome and gene function analysis with the PANTHER classification system (v.14.0). Nat. Protoc. 14, 703-721. doi: 10.1038/ s41596-019-0128-8

Ming, X., Pirtskhalava, T., Farr, J. N., Weigand, B. M., Palmer, A. K., Weivoda, M. M., et al. (2018). Senolytics improve physical function and increase lifespan in old age. Nat. Med. 24, 1246-1256. doi: 10.1038/s41591-018-0092-9

Oda, T., Sakai, T., Hiraiwa, H., Hamada, T., Ono, Y., Nakashima, M., et al. (2016). Osteoarthritis-derived chondrocytes are a potential source of multipotent progenitor cells for cartilage tissue engineering. Biochem. Biophys. Res. Co. 479, 469-475. doi: 10.1016/j.bbrc.2016.09.085

Ragan, P. M., Chin, V. I., Hung, H., Masuda, K., Thonar, J., Arner, E. C., et al. (2000). Chondrocyte extracellular matrix synthesis and turnover are influenced by static compression in a new alginate disk culture system. Arch. Biochem. Biophys. 383, 256-264. doi: 10.1006/abbi.2000.2060

Robinson, W. H., Lepus, C. M., Wang, Q., Raghu, H., Mao, R., Lindstrom, T. M., et al. (2016). Low-grade inflammation as a key mediator of the pathogenesis of osteoarthritis. Nat. Rev. Rheumatol. 12, 580-592. doi: 10.1038/nrrheum.2016. 136

Sivan, S. S., Wachtel, E., Tsitron, E., Sakkee, N., van der Ham, F., Degroot, J., et al. (2008). Collagen turnover in normal and degenerate human intervertebral discs as determined by the racemization of aspartic acid. J. Biol. Chem, 283, 8796-8801. doi: 10.1074/jbc.M709885200

Subramanian, A., Tamayo, P., Mootha, V. K., Mukherjee, S., Ebert, B. L., Gillette, M. A., et al. (2005). Gene set enrichment analysis: a knowledge-based approach for interpreting genome-wide expression profiles. Proc. Natl. Acad. Sci. U.S.A. 102, 15545-15550. doi: 10.1073/pnas.0506580102

Tchkonia, T., Zhu, Y., Deursen, J. V., Campisi, J., and Kirkland, J. L. (2013). Cellular senescence and the senescent secretory phenotype: therapeutic opportunities. J. Clin. Invest. 123, 966-972. doi: 10.1172/JCI64098

Vozzi, G., Lucarini, G., Dicarlo, M., Andreoni, C., Salvolini, E., Ferretti, C., et al. (2016). In vitro lifespan and senescent behaviour of human periosteal derived stem cells. Bone 88, 1-12. doi: 10.1016/j.bone.2016.04.013

Wajant, H., and Siegmund, D. (2019). TNFR1 and TNFR2 in the control of the life and death balance of macrophages. Front. Cell Dev. Biol. 7:91. doi: 10.3389/fcell. 2019.00091

Xie, C., and Chen, Q. (2019). Adipokines: new therapeutic target for osteoarthritis? Curr. Rheumatol. Rep. 21:71. doi: 10.1007/s11926-019-0868-z

Xu, M., Bradley, E. W., Weivoda, M. M., Hwang, S. M., Pirtskhalava, T., Decklever, T., et al. (2017). Transplanted senescent cells induce an osteoarthritis-like condition in mice. J. Gerontol. A Biol. Sci. Med. Sci. 72, 780-785. doi: 10.1093/ gerona/glw154

Conflict of Interest: CJ and QC have pending patent applications of generating OA-MSC cell lines. OA-MSC cell lines have been licensed to Applied Biological Materials Inc.

The remaining authors declare that the research was conducted in the absence of any commercial or financial relationships that could be construed as a potential conflict of interest.

Publisher's Note: All claims expressed in this article are solely those of the authors and do not necessarily represent those of their affiliated organizations, or those of the publisher, the editors and the reviewers. Any product that may be evaluated in this article, or claim that may be made by its manufacturer, is not guaranteed or endorsed by the publisher.

Copyright (๑) 2021 Liu, Brodsky, Feng, Liu, Ding, Jayasuriya and Chen. This is an open-access article distributed under the terms of the Creative Commons Attribution License (CC BY). The use, distribution or reproduction in other forums is permitted, provided the original author(s) and the copyright owner(s) are credited and that the original publication in this journal is cited, in accordance with accepted academic practice. No use, distribution or reproduction is permitted which does not comply with these terms. 